\title{
Resonance-Enhanced Raman Spectroscopy on Explosives Vapor at Standoff Distances
}

\author{
Anneli Ehlerding, Ida Johansson, Sara Wallin, and Henric Östmark \\ Department of Energetic Materials, Swedish Defense Research Agency (FOI), 14725 Tumba, Sweden \\ Correspondence should be addressed to Anneli Ehlerding, anneli.ehlerding@foi.se
}

Received 23 June 2011; Revised 19 September 2011; Accepted 2 November 2011

Academic Editor: S. Michael Angel

Copyright ( $) 2012$ Anneli Ehlerding et al. This is an open access article distributed under the Creative Commons Attribution License, which permits unrestricted use, distribution, and reproduction in any medium, provided the original work is properly cited.

\begin{abstract}
Resonance-enhanced Raman spectroscopy has been used to perform standoff measurements on nitromethane (NM), 2,4-DNT, and 2,4,6-TNT in vapor phase. The Raman cross sections for NM, DNT, and TNT in vapor phase have been measured in the wavelength range $210-300 \mathrm{~nm}$ under laboratory conditions, in order to estimate how large resonance enhancement factors can be achieved for these explosives. The results show that the signal is enhanced up to 250,000 times for 2,4-DNT and up to 60,000 times for 2,4,6-TNT compared to the nonresonant signal at $532 \mathrm{~nm}$. Realistic outdoor measurements on NM in vapor phase at $13 \mathrm{~m}$ distance were also performed, which indicate a potential for resonance Raman spectroscopy as a standoff technique for detection of vapor phase explosives. In addition, the Raman spectra of acetone, ethanol, and methanol were measured at the same wavelengths, and their influence on the spectrum from NM was investigated.
\end{abstract}

\section{Introduction}

Improvised explosive devices (IEDs) are a common and a growing threat to the civilian society as well as to peace-keeping operations. Today, detection and identification of explosive charges and IEDs in real environments (indoors or outdoors) is a very challenging task and can in many cases also be a very hazardous operation. IEDs are in a way unique compared to regular ordnances, since the IED manufacturer has to improvise the design and use whatever materials are available. For this reason, the threat varies over time and in different parts of the world; it also evolves as countermeasures are taken. The diverse and varying nature of IEDs poses great challenges to the methods for their detection since they can contain any explosive which is available; this means commercial, military, or homemade explosives (HMEs). An overview of HME properties is given by Menning and Ostmark [1]. From the perspective of the user of the detection equipment, much would be gained if detection and identification could be reliably performed from a safe distance. This drives the research of possible standoff detection methods, where the developed methods will need to be both sensitive and selective in order to detect an explosive material in vapor phase and at low concentrations, or as trace amounts of particles on a surface, among an unknown number and composition of possible interfering substances. In addition, the method should be applicable at a distance of some tenths to a few hundreds of meters.

Techniques that are anticipated to fulfill these requirements are laser-based spectroscopy methods. A number of different laser-based methods for stand-off detection and identification of explosives have been proposed and tested, for example, Raman, LIBS, and PF/LIF. For reviews see Wallin et al. [2] and Gottfried et al. [3]. Raman scattering is an instantaneous inelastic scattering of photons where some energy is lost to (or gained from) the target molecule, returning scattered light with a different wavelength. The difference in energy corresponds to a vibrational mode of the target molecules, and these vibrational modes can be regarded as a fingerprint that uniquely identifies the substance or substances in a sample. Also complex mixtures can often be analyzed using algorithms for pattern recognition [4].

Standoff Raman explosives detection systems have been developed and tested by a number of groups; Sharma et al. demonstrated explosives detection (TATB and HMX) at $10 \mathrm{~m}$ distance using a frequency-doubled Nd:YAG laser 
[5]. Detection of diluted explosives in a silicon matrix at longer distances, $27 \mathrm{~m}$ and $50 \mathrm{~m}$, was demonstrated by Carter et al. in a laboratory environment using a frequency doubled Nd:YAG and gated detection [6]. The usefulness of spontaneous Raman spectroscopy for stand-off detection in more realistic scenarios has also been demonstrated $[7,8]$. The instrumentation used was based on a frequency doubled pulsed Nd:YAG laser, a Schmidt-Cassegrain telescope, a spectrometer for wavelength dispersion, and an ICCD for gated detection. These measurements showed the applicability of $532 \mathrm{~nm}$ radiation for examination through different container materials (transparent or semitransparent) and during varying weather conditions at distances of up to $55 \mathrm{~m}$. Recent results using this equipment have shown that the detection limit for, for example, TNT at $30 \mathrm{~m}$ and a detection time of $5 \mathrm{~s}$ is approximately $500 \mu \mathrm{g}[4,9]$. This indicates the difficulties in using conventional Raman for stand-off trace and vapor detection.

Several groups have pursued Raman spectroscopy at UV wavelengths. The reasons for doing so are multiple; the obvious one is that the Raman signal intensity scales as $1 / \lambda^{4}$, thus leading to an improvement of the inherently weak Raman response upon examination. Another advantage is the much reduced interference of fluorescence [10]. At wavelengths shorter than $250 \mathrm{~nm}$, the full Raman range, up to $4,000 \mathrm{~cm}^{-1}$, is normally free from luminescence. This can be used for identifying species in a fluorescent matrix [11]. If a laser wavelength outside the visible spectral region is used, the problem of eye hazards is significantly decreased since the maximum permissible exposure is markedly higher for wavelengths that are not focused by the lens of the human eye onto the retina. Of all the advantages of turning to UV wavelengths, perhaps the most intriguing, is the possible resonance enhancement that can occur when the probe laser wavelength is nearing an electronic transition within the molecule. This can give rise to enhancements of certain Raman lines (those coupled to the electronic transition) for which the Raman cross section can be significantly enhanced by a factor of $10^{2}-10^{6}$ [12]. A number of Raman publications have had a focus on resonance-enhanced UV Raman spectroscopy of explosives [11, 13, 14]. Absolute Raman cross sections for UN, TATP, RDX, TNT, and PETN at $248 \mathrm{~nm}$ excitation have been published by Nagli et al. [13], and for TNT, PETN, RDX, and HMX at $229 \mathrm{~nm}$ excitation by Tuschel et al. [14].

The ability to detect explosives in vapor phase depends on the vapor pressure of the explosive. Some explosives, such as triacetone triperoxide (TATP), have a relatively high vapor pressure $\left(4.65 \times 10^{-2}\right.$ Torr at $\left.25^{\circ} \mathrm{C}[15]\right)$ and can be expected to be found in concentrations detectable also with methods with high detection limits $\left(4.65 \times 10^{-2}\right.$ Torr corresponds to $60 \mathrm{ppm}$ at atmospheric pressure) whereas other explosives, such as HMX, have very low vapor pressure [16] and can probably not be expected to be detectable with any gas phase detection methods. In this paper we have measured the Raman signal and calculated the apparent Raman cross section in the UV region, which is the cross section based on the measured signal without taking into account the effects of absorption and self-absorption. The focus is on investigating if resonance-enhanced Raman spectroscopy could be used as a stand-off explosives detection method, and absorption and self-absorption do not have a large influence on that application. We have focused on nitromethane (NM), 2,4DNT and 2,4,6-TNT. We have also studied the Raman spectra of a few solvents: acetone, ethanol, and methanol, to investigate their influence on the Raman spectrum of nitromethane.

\section{Experiment}

Measurements were performed both in laboratory settings with controlled ambient conditions and outdoors; the measurement setups are schematically described in Figure 1. The investigated substances were nitromethane (NM) $(>98 \%)$, 2,4-DNT ( $>97 \%$ ), and 2,4,6-TNT. Pure 2,4,6-TNT was obtained by dissolving military grade TNT in ethylacetate, washing three times with a $5 \%$ aqueous solution of $\mathrm{NaHSO}_{3}$, drying the remaining organic phase over $\mathrm{Na}_{2} \mathrm{SO}_{4}$ and recrystallizing the solid residue once from $99.5 \%$ ethanol. The white crystals were dried at $40^{\circ} \mathrm{C}$ overnight. In addition, the solvents acetone (>99\%), ethanol (99.7\%), and methanol (99.8\%) were studied.

The explosives vapor was generated from a liquid or solid sample placed on a Petri dish. For the measurements using the vapor chamber, the Petri dish was placed inside the chamber, nitrogen (AGA, OTC-50) was added to reach atmospheric pressure, and equilibrium was allowed to be established. Equilibrium was decided as being when the signal from the sample did not increase with time; this process required different amount of time for different samples. When measuring on a combination of substances (NM and solvent), they were placed on two separate Petri dishes. The concentration $c(T)$ of the vaporized explosive in the chamber was determined by the ratio of the vapor pressure of the sample, $P_{v, \mathrm{HE}}(T)$, and the total pressure in the chamber, $P_{\text {total }}$ as

$$
c(T)=\frac{N_{\mathrm{HE}}(T)}{N_{\text {total }}}=\frac{P_{v, \mathrm{HE}}(T)}{P_{\text {total }}},
$$

where $N_{\mathrm{HE}}(T)$ is the number of molecules of the sample in gas phase and $N_{\text {total }}$ the total number of gas molecules in the chamber. The vapor pressure of NM has been taken from McCullough et al. [17], the vapor pressure of 2,4-DNT from Pella [18] and of 2,4,6-TNT from Edwards [19]. The vapor chamber (Al, $340 \mathrm{~mm}$ long, $\varnothing 200 \mathrm{~mm}$ ) has a high UVtransmitting window on each side (10 mm uncoated UV-FS). In the measurements on DNT and TNT, the temperature of the chamber was elevated to above the melting point of the samples in order to reach a high enough concentration while the temperature and pressure were continuously monitored. To avoid condensation of explosives on the windows, they were held at a higher temperature than the rest of the chamber. When changing the sample, the chamber was cleaned and heated to $80^{\circ} \mathrm{C}$ overnight, while evacuating the system. In the measurements without the vapor chamber the sample was put outside the laboratory on a Petri dish and 


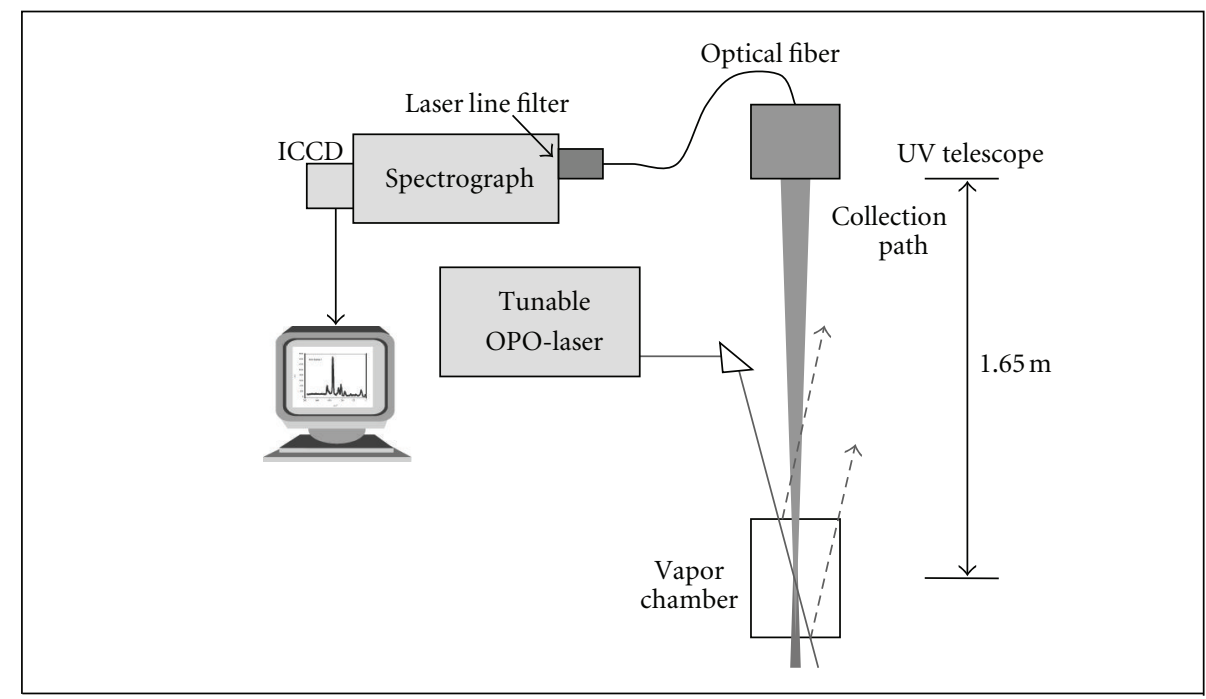

(a)

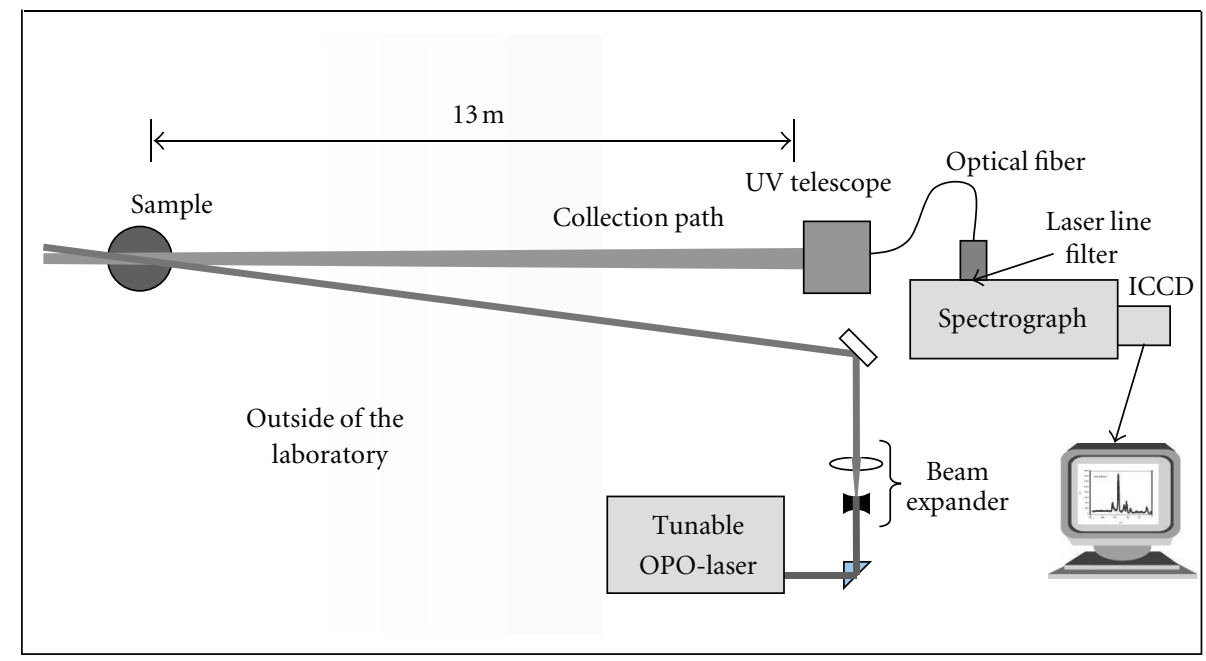

(b)

FIGURE 1: Schematic picture of the resonant Raman spectroscopy setup (a) using the vapor chamber and (b) outdoors in open air.

heated to about $328 \mathrm{~K}$ while the surrounding air temperature was around 274-278 K.

A tunable Optical Parametric Oscillator (OPO), pumped by the 3rd harmonic of a Nd:YAG laser (pulse length $6 \mathrm{~ns}$, repetition rate $10 \mathrm{~Hz}$, pulse energy 2-5 $\mathrm{mJ}$ ), was used in the measurements, and the beam was directed towards the chamber via a right-angle prism (UV-FS). The signal was measured in a back scatter configuration, where the scattered light was collected by a 5 " UV telescope at a stand-off distance of 1.65 meters. The telescope was focused at the centre of the chamber where it collected light from a path with a width of $8 \mathrm{~mm}$. The diameter of the laser beam was approximately $5-8 \mathrm{~mm}$, depending on the wavelength. No expansion of the laser beam was used. The laser beam and the collection path of the telescope overlapped only inside the chamber, and thus the Raman signal was only collected from a volume (approximately $4 \mathrm{~cm}^{3}$ ) where the explosive vapor concentration in relation to the nitrogen concentration was known. Nitrogen has a known Raman cross section [20] and is known not to undergo resonance enhancement in the spectral region of interest in these measurements. It was therefore used as an internal reference. Measurements performed without nitrogen in the chamber showed that the possible contribution to the nitrogen peak from air outside the chamber was negligible.

For the measurements outdoors on NM without the vapor chamber, the same OPO laser and equipment was used, but the laser beam was expanded 4-5 times and the standoff distance was 13 meters. The beam was directed out from the laboratory by a mirror to coincide with the collection path of the telescope about $5 \mathrm{~cm}$ above the sample. Hence, the setup had an oblique configuration where the path of the laser beam and the collection path of the telescope were crossed only for a limited spatial section. The diameter of 
the collection path of the telescope was approximately $55 \mathrm{~mm}$ at the sample, which was slightly larger than the laser beam diameter.

The light was introduced into a spectrometer via an optical round-to-slit fiber bundle $(450 \mu \mathrm{m}$ diameter at the round end). The spectrometer was a USB-connected, computer controlled $500 \mathrm{~mm}$ Czerny-Turner spectrometer with a 2400 groves $/ \mathrm{mm}$ grating, resulting in a covered spectral range of around $1000-3000 \mathrm{~cm}^{-1}$, which is sufficient to see first order resonant peaks of the investigated substances and the nitrogen peaks for UV wavelengths. The Raman spectra were registered by an ICCD camera with nanosecond gating time. The camera was held at $-20^{\circ} \mathrm{C}$. All measurements shown in this paper are accumulated during 1000 laser pulses, that is, 100 seconds.

Laser line long pass filters were used in the 2,4,6-TNT measurements and in the 2,4-DNT measurements in order to reduce the background from stray laser light. The Raman signal from TNT was measured for incident wavelengths between $243 \mathrm{~nm}$ and $268 \mathrm{~nm}$. Since a Raman shift of $1350 \mathrm{~cm}^{-1}$ (around the lowest Raman shift observed for TNT in these measurements) corresponds to a shift of around 8-9 $\mathrm{nm}$ for these wavelengths, the filters were used also for incident laser wavelengths below the filter cutoff, and three different laser line filters were used to cover the whole range of Raman scattered signal. A $248 \mathrm{~nm}$ filter was used for incident wavelengths in the range $243-250 \mathrm{~nm}$, a $257 \mathrm{~nm}$ filter in the range $251-258 \mathrm{~nm}$, and a $266 \mathrm{~nm}$ filter in the range 260 $268 \mathrm{~nm}$. The transmission of the filters drops somewhat at the lower wavelengths in the range, and corrections were performed to account for this using the filter data from the manufacturer.

The response to UV light of the setup was investigated by measuring the spectrum from a calibrated UV-light source. The response of the system decreases rapidly at smaller wavelengths. However, measuring the resonant Raman signal for pure $\mathrm{N}_{2}$ for an excitation wavelength of $210 \mathrm{~nm}$ results in a clearly detectable Raman shifted signal at $215 \mathrm{~nm}$. Thus, despite the low response to wavelengths below $230 \mathrm{~nm}$, the sensitivity of the system still enables detection in this range.

\section{Data Analysis}

The areas of the Raman peaks from the NM, DNT, and TNT, $I_{\mathrm{HE}}(\lambda)$, and from $\mathrm{N}_{2}, I_{\mathrm{N}_{2}}(\lambda)$, were extracted from the measured spectra (examples of spectra are shown in Figure 2) after subtracting the background noise level. From this measured signal and the pressure and temperature of the vapor chamber, the enhancement factors and measured cross sections were calculated. Nitrogen is used as an internal reference, and the Raman cross section of $\mathrm{N}_{2}, \sigma_{\mathrm{N}_{2}}(\lambda)$, is used for normalization. The Raman cross section for nitrogen behaves nonresonant in the wavelength range $210-300 \mathrm{~nm}$ and can thus be extrapolated from the value of $3.5 \times$ $10^{-30} \mathrm{~cm}^{2} \mathrm{str}^{-1}$ at $337 \mathrm{~nm}$ [20]. The Raman cross sections of the studied substance, $\sigma_{\mathrm{HE}}(\lambda)$, can then be expressed as

$$
\sigma_{\mathrm{HE}}(\lambda)=\sigma_{\mathrm{N}_{2}}(\lambda) \frac{I_{\mathrm{HE}}(\lambda)}{I_{\mathrm{N}_{2}}(\lambda)} \frac{N_{\mathrm{N}_{2}}}{N_{\mathrm{HE}}} A_{\mathrm{abs}}\left(\lambda, N_{\mathrm{HE}}\right),
$$

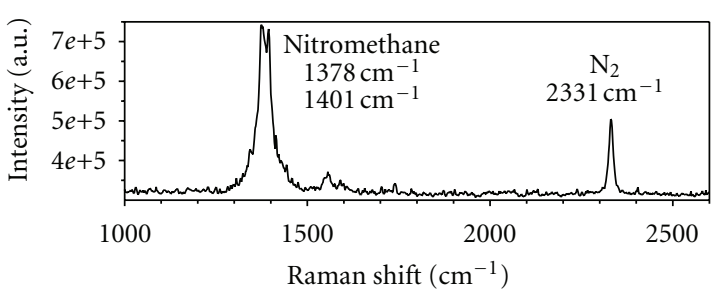

(a)

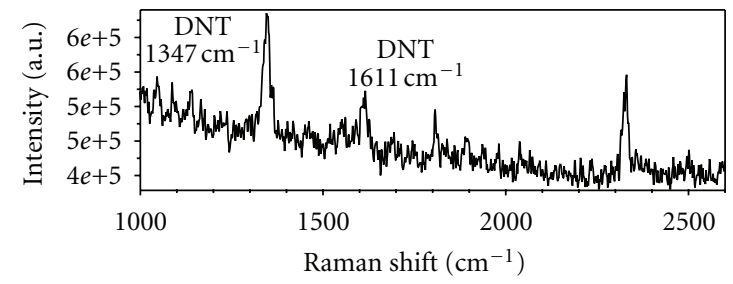

(b)

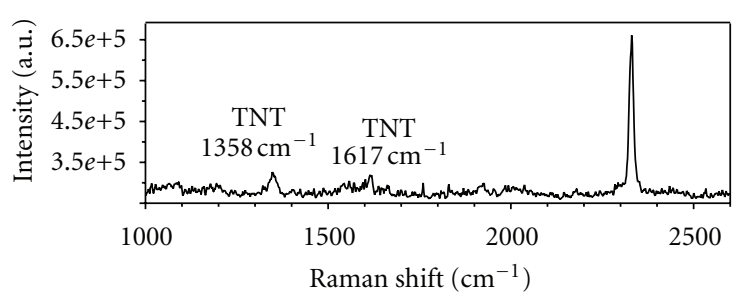

(c)

FIGURE 2: Resonant Raman signal from (a) NM at $232 \mathrm{~nm}$ excitation wavelength, concentration $32,500 \mathrm{ppm}$, (b) 2,4-DNT at $263 \mathrm{~nm}$, concentration $1445 \mathrm{ppm}$, and (c) 2,4,6-TNT at $248 \mathrm{~nm}$, concentration $341 \mathrm{ppm}$. All spectra are recorded during 1000 laser pulses, that is, $100 \mathrm{~s}$, at a distance of $1.65 \mathrm{~m}$. The TNT spectrum was recorded using a $248 \mathrm{~nm}$ long-pass filter, hence reducing the noise level.

where $N_{\mathrm{N}_{2}}$ and $N_{\mathrm{HE}}$ are extracted from (1), and $A_{\mathrm{abs}}\left(\lambda, N_{\mathrm{HE}}\right)$ takes into account the change in signal due to absorption and self-absorption. The effect of this will be discussed later however, in this paper we have not been able to assess these parameters, but focus has been on demonstrating the resonance enhancement. Therefore, (2) has been simplified to calculate the apparent cross section, $\sigma_{\mathrm{HE}, \text { app }}$, which is the Raman cross section where the effect of absorption and selfabsorption is not taken into account:

$$
\sigma_{\mathrm{HE}, \text { app }}(\lambda)=\sigma_{\mathrm{N}_{2}}(\lambda) \frac{I_{\mathrm{HE}}(\lambda)}{I_{\mathrm{N}_{2}}(\lambda)} \frac{N_{\mathrm{N}_{2}}}{N_{\mathrm{HE}}} .
$$

The apparent Raman enhancement factor compared to the Raman signal at $532 \mathrm{~nm}, F_{532}(\lambda)$, can then be calculated as

$$
F_{532}(\lambda)=\frac{\sigma_{\mathrm{HE}, \mathrm{app}}(\lambda)}{\sigma_{\mathrm{HE}}(532)} .
$$

The cross section at $532 \mathrm{~nm}$ for NM could not be found in the literature. Therefore, the Raman activities of NM and $\mathrm{N}_{2}$ were calculated using Gaussian [21] (HF 6-31G(d,p)) and used to scale the Raman cross section for $\mathrm{N}_{2}$ to give the cross section for NM. The Raman cross section for $\mathrm{NM}$ at $532 \mathrm{~nm}$ was found to be $3.65 \times 10^{-31} \mathrm{~cm}^{2} \mathrm{str}^{-1}$ and $3.79 \times 10^{-31} \mathrm{~cm}^{2} \mathrm{str}^{-1}$ for the $1378 \mathrm{~cm}^{-1}$ and $1401 \mathrm{~cm}^{-1}$ lines, 
respectively. The Raman cross section for the $1358 \mathrm{~cm}^{-1}$ line in TNT is $4.9 \times 10^{-31} \mathrm{~cm}^{2} \mathrm{str}^{-1}$ [22]. The Raman cross section for DNT has not to our knowledge been published, and we estimate the cross section of the $1347 \mathrm{~cm}^{-1}$ line in DNT to be the same as the cross section for the $1358 \mathrm{~cm}^{-1}$ line in TNT at $532 \mathrm{~nm}$.

\section{Results and Discussions}

4.1. Resonance-Enhanced Raman Cross Sections. Spectra from vapor phase resonance Raman measurements on NM, 2,4-DNT and 2,4,6-TNT in the vapor chamber were recorded at several wavelengths in the range $210-290 \mathrm{~nm}$. Examples of the spectra are shown in Figure 2, showing measurements performed at an incident wavelength of $232 \mathrm{~nm}, 263 \mathrm{~nm}$, and $248 \mathrm{~nm}$ for NM, DNT, and TNT, respectively, which is close to the observed maximum in the resonant Raman enhancement for each substance. However, as will be discussed below, the enhancement might be higher at lower wavelengths. In the displayed spectra, the background level has not been subtracted. The NM and DNT spectra in Figure 2 were recorded without the use of laser line filters, whereas for TNT a $248 \mathrm{~nm}$ long-pass filter was used.

The NM spectrum, Figure 2(a), shows a clear enhancement of the $1378 \mathrm{~cm}^{-1}$ and $1401 \mathrm{~cm}^{-1}$ lines. The resolution of the system does not allow them to be completely resolved and they are seen as a double peak. The signal at $2331 \mathrm{~cm}^{-1}$ is the signal from $\mathrm{N}_{2}$. The measurement on $\mathrm{NM}$ was performed at room temperature, $291 \mathrm{~K}$, and the vapor pressure was 24.35 Torr [17]. The atmospheric pressure varied between 744 Torr and 750 Torr during the measurements, resulting in a NM concentration between 32,500 and $32,700 \mathrm{ppm}$. The 2,4-DNT spectrum shown in Figure 2(b) exhibits an enhancement of the $1347 \mathrm{~cm}^{-1}$ and $1611 \mathrm{~cm}^{-1}$ lines, and for 2,4,6-TNT, correspondingly, the $1358 \mathrm{~cm}^{-1}$ and $1617 \mathrm{~cm}^{-1}$ lines were enhanced, as seen in Figure 2(c). DNT and TNT were heated in order to reach measurable concentrations; in the measurements presented in Figure 2, the temperature was $110^{\circ} \mathrm{C}$ and $128^{\circ} \mathrm{C}$ for DNT and TNT, respectively, resulting in concentrations of $1445 \mathrm{ppm}$ DNT and $341 \mathrm{ppm}$ TNT.

For each spectrum, the integrated signal of the $1378 \mathrm{~cm}^{-1}$ and $1401 \mathrm{~cm}^{-1}$ lines from NM, the $1347 \mathrm{~cm}^{-1}$ line from DNT and the $1358 \mathrm{~cm}^{-1}$ line from TNT was used to calculate the apparent Raman cross section, as described in (3). The apparent Raman cross sections as a function of incident wavelength are shown in Figures 3-5. The wavelength of maximum resonance enhancement should coincide approximately with an absorption line in the sample. In order to correlate the cross sections at different wavelengths with the absorption lines, the UV-absorption spectra of NM, DNT, and TNT were measured in vapor phase using a UV/visible spectrometer, and the results are shown in Figure 6. It should be noted that the absorption peaks could contain multiple absorption lines.

The arrows in Figure 6 indicate where the maxima in apparent Raman cross section are found in vapor phase: for NM at $232 \mathrm{~nm}$, for DNT between $252 \mathrm{~nm}$, and $258 \mathrm{~nm}$ and for TNT around $250 \mathrm{~nm}$. Approaching an absorption

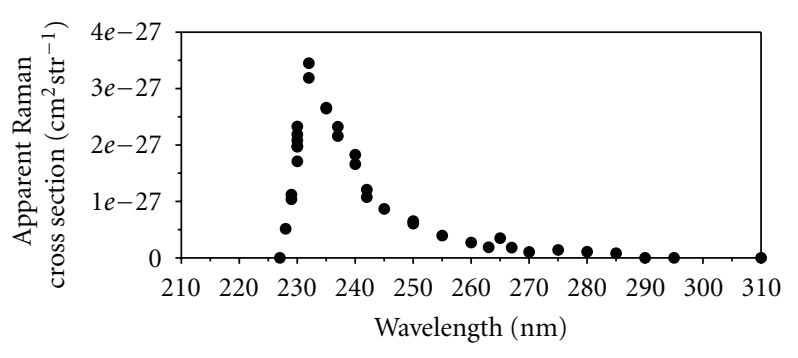

FIGURE 3: The apparent Raman cross section for the Raman shifted signal at $1378 \mathrm{~cm}^{-1}$ and $1401 \mathrm{~cm}^{-1}$ in NM as a function of incident wavelength. The concentration of NM was between 32,500 and $32,700 \mathrm{ppm}$ at room temperature, $291 \mathrm{~K}$.

peak, absorption and self-absorption are likely to occur in the measured Raman signal, resulting in a reduced signal as explained in Section 3. A large part of the photons from the laser will be absorbed in the vapor before reaching the center of the chamber where the Raman signal is detected. This is believed to be mainly what happens, for example below $230 \mathrm{~nm}$ in the NM measurements, where the Raman signal, also from $\mathrm{N}_{2}$, entirely disappears. Transmission measurements of the laser light through the vapor chamber also show a sharp drop in transmittance between $230 \mathrm{~nm}$ and $240 \mathrm{~nm}$, which support this interpretation. Continuing the example of NM, at wavelengths around $230 \mathrm{~nm}$ and above, the NM molecules will be illuminated by laser light, but they will also have a high probability to self-absorb the scattered light. This probability increases with the sample concentration in the chamber. The apparent cross section therefore gives a minimum value, and the absolute cross section is in fact higher than this. A third aspect which might contribute to the drop in signal is the weaker system response at low wavelengths. This is, however, expected to be a smaller issue than absorption and self-absorption.

For 2,4-DNT, the Raman signal was measured at four different temperatures, that is, concentrations, in the wavelength range from $220 \mathrm{~nm}$ to $280 \mathrm{~nm}$. The apparent cross sections for the Raman shifted signal at $1347 \mathrm{~cm}^{-1}$ are shown in Figure 4. In the measurements of the wavelength-dependent signal of DNT, no laser-line filters were used. As seen, the apparent cross sections measured at different concentrations differ in both magnitude and wavelength for maximum cross section; at lower concentrations it is higher and the maximum tends to be shifted to shorter wavelengths, that is, closer to the absorption peak. The absolute cross section is of course the same independent of the concentration, and the observed change in magnitude is due to absorption and selfabsorption in DNT in the same way as for NM. Similarly, the fact that the maximum in the apparent Raman cross section is slightly shifted towards lower wavelengths and increased in magnitude at lower concentrations indicates lower absorption at lower concentrations. Transmission measurements of the laser light through the vapor chamber shows a clear drop in transmission at around $230-260 \mathrm{~nm}$, indicating that the absorption is strong enough to be important.

The wavelength dependence of the Raman signal of 2,4,6TNT was only investigated at a concentration of $341 \mathrm{ppm}$, 


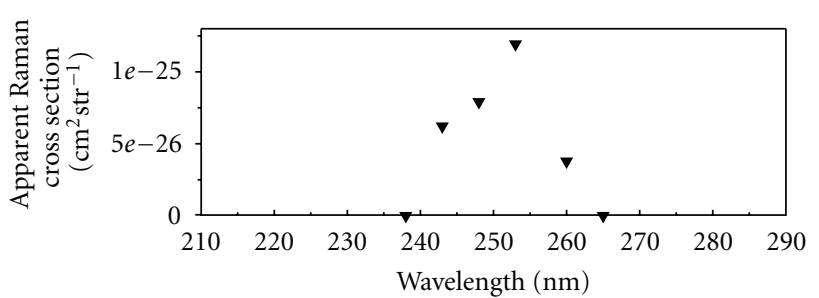

(a)

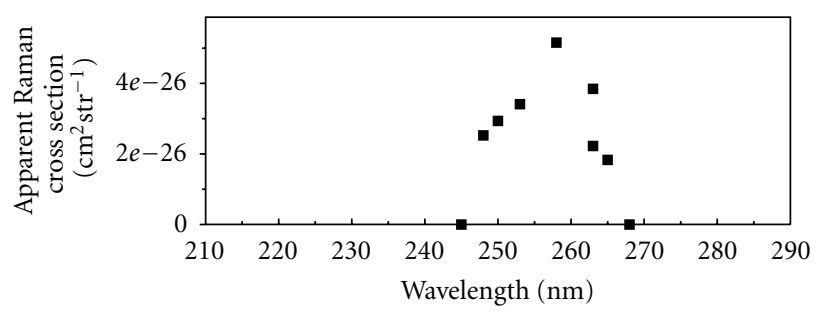

(b)

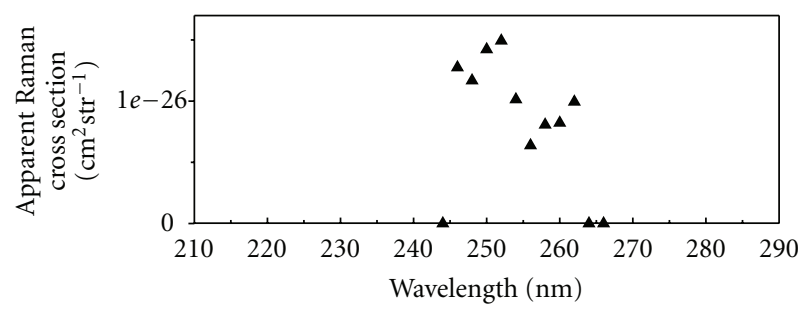

(c)

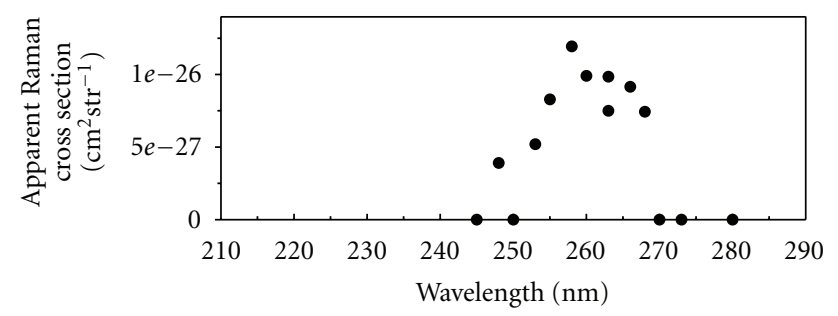

(d)

FIgURE 4: The apparent Raman cross section for the Raman shifted signal at $1347 \mathrm{~cm}^{-1}$ in DNT as a function of incident wavelength at (a) $354 \mathrm{~K}$, concentration $126 \mathrm{ppm}$, (b) $368 \mathrm{~K}$, concentration $432 \mathrm{ppm}$, (c) $371 \mathrm{~K}$, concentration $550 \mathrm{ppm}$, and (d) $383 \mathrm{~K}$, concentration $1445 \mathrm{ppm}$.

and the apparent cross section was not measured in the wavelength region below $243 \mathrm{~nm}$.

4.2. Enhancement Compared to $532 \mathrm{~nm}$ and the Dependence on Concentration. The apparent Raman cross sections for NM, DNT, and TNT presented in Figures 3-5 have been used in (4) to estimate the resonance enhancement compared to the Raman cross section at $532 \mathrm{~nm}$ for each substance. The Raman cross section for DNT was assumed to be the same as the cross section for TNT at $532 \mathrm{~nm}$, that is, $4.9 \times$ $10^{-31} \mathrm{~cm}^{2} \mathrm{str}^{-1}$. For all three substances a strong enhancement is seen, and the apparent enhancement factors $F_{\max }$ are shown in Table 1.

The DNT resonance enhancement increases with one order of magnitude as the concentration is reduced about the

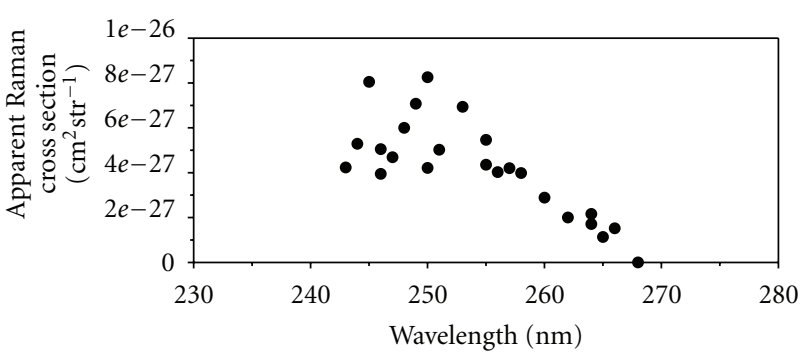

Figure 5: The apparent Raman cross section of the Raman shifted signal at $1358 \mathrm{~cm}^{-1}$ in TNT as a function of incident wavelength at $401 \mathrm{~K}$, TNT concentration $341 \mathrm{ppm}$.

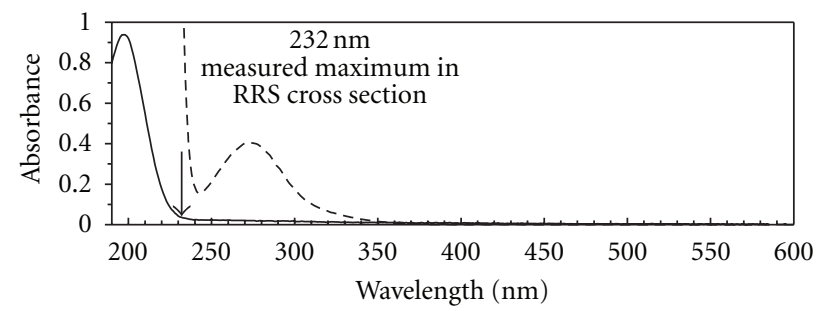

(a)

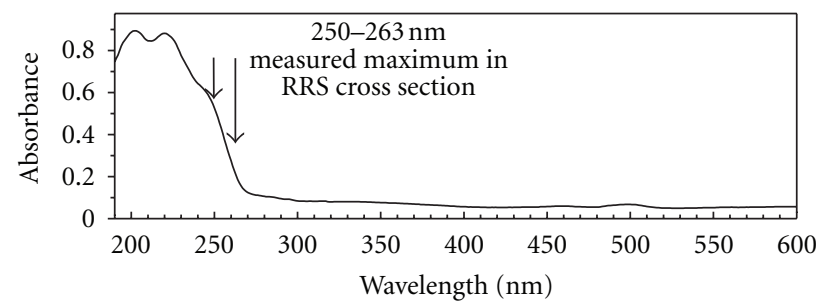

(b)

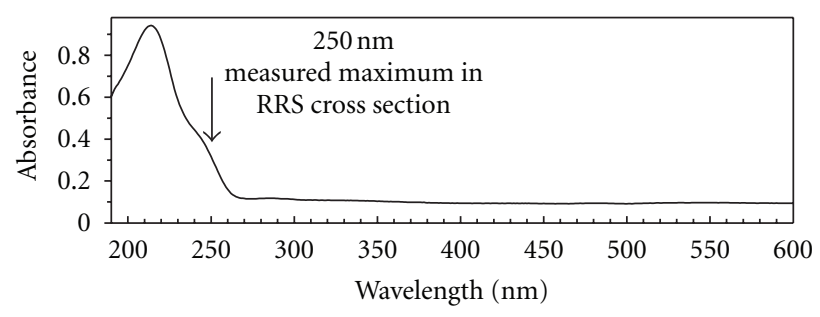

(c)

FIGURE 6: UV-absorption spectra of (a) NM, (b) 2,4-DNT, and (c) 2,4,6-TNT in vapor phase, measured with a UV/visible absorption spectrometer. The dashed line in (a) is a measurement with higher NM concentration, revealing an absorption maximum at $273 \mathrm{~nm}$. The absolute sample concentrations were not measured. The arrows show the wavelengths where the maximum in apparent resonant Raman cross section is found in the vapor phase measurements of each substance. The UV spectra were taken at $291 \mathrm{~K}, 353 \mathrm{~K}$, and $393 \mathrm{~K}$ for NM, DNT, and TNT, respectively.

same factor, and although the signal at $354 \mathrm{~K}$ was very weak and embedded in the noise, which is why the temperature was raised to higher values, it is still clear that a stronger enhancement is measured at lower concentrations where the absorption is less.

The apparent Raman cross section of TNT at $248 \mathrm{~nm}$ excitation is one order of magnitude lower than the absolute 
TABle 1: Apparent maximum enhancements, $F_{\max }$, compared to the Raman cross section at $532 \mathrm{~nm}$ for NM, DNT, and TNT at the given concentrations. $\lambda_{\max }$ is the incident wavelength resulting in the highest cross section, $\omega$ is the Raman wave number of the observed Raman peak, and $\sigma_{\mathrm{HE} \text {,app }}$ is the apparent Raman cross section. The Raman signal for TNT at a concentration of $26 \mathrm{ppm}$ is only measured at $248 \mathrm{~nm}$; a higher value might be found at another wavelength. The apparent cross section is included in the table to facilitate comparison with other measurements and should not be mistaken for an absolute Raman cross section.

\begin{tabular}{|c|c|c|c|c|c|c|}
\hline Substance & $\lambda_{\max }(\mathrm{nm})$ & $\omega\left(\mathrm{cm}^{-1}\right)$ & $\sigma_{\mathrm{HE}, \text { app }}\left(\mathrm{cm}^{2} \mathrm{str}^{-1}\right)$ & Temp. (K) & Conc. (ppm) & $F_{\max }$ \\
\hline $\mathrm{NM}$ & 232 & $1378 / 1401$ & $3.4 \times 10^{-27}$ & 291 & 32,500 & 9,300 \\
\hline 2,4-DNT & 262 & 1347 & $1 \times 10^{-26}$ & 383 & 1,445 & 24,000 \\
\hline 2,4-DNT & 252 & 1347 & $1 \times 10^{-25}$ & 354 & 126 & 240,000 \\
\hline $2,4,6-\mathrm{TNT}$ & 250 & 1358 & $8 \times 10^{-27}$ & 401 & 341 & 17,000 \\
\hline 2,4,6-TNT & 248 & 1358 & $3 \times 10^{-26}$ & 369 & 26 & 61,000 \\
\hline
\end{tabular}

cross section published for TNT in solution at an incident wavelength of $229 \mathrm{~nm}$ by Tuschel et al. [14]. Since the apparent cross sections presented here are believed to be reduced by absorption and to in fact increase at lower wavelength, this could account for the discrepancy. However, it cannot be assumed that the Raman cross section should be identical for solution and vapor phase samples. Absolute Raman solid-state cross sections of TNT for an incident wavelength of $248 \mathrm{~nm}$ reported by Nagli et al. [13], however, are around two orders of magnitude lower than the present apparent cross section. This is a surprisingly large difference for which we have no clear explanation; possible reasons could be absorption issues also in the solid-state experiments or discrepancies between the solid-state and vapor Raman cross sections.

The dependence on the concentration for the resonance Raman signal was investigated for 2,4-DNT and 2,4,6-TNT in the concentration range $10-1445 \mathrm{ppm}$ and $26-364 \mathrm{ppm}$, respectively. This measurement was performed to get an estimate of the influence of self-absorption and investigate how the resonant behavior could be explored in an application. Without the absorption, factor the resonance enhancement is independent of the sample concentration. The resonance Raman signal for incident light of $248 \mathrm{~nm}$ was systematically monitored using a $248 \mathrm{~nm}$ long-pass filter while heating the chamber, that is, increasing the concentration. The apparent enhancement of the resonance Raman signal compared to the Raman cross section at $532 \mathrm{~nm}$ was then calculated for different concentrations and is shown in Figure 7.

4.3. Measurements on Solvents at $232 \mathrm{~nm}$. The results from measurements on acetone, ethanol and methanol are presented in Figures 8, 9, and 10. The upper spectra were recorded for the pure solvents and the lower spectra were recorded for the solvent together with NM. The spectra were taken using a laser wavelength of $232 \mathrm{~nm}$, which is where the maximum signal from NM was observed at room temperature. All solvent measurements were performed at room temperature. During the pure acetone measurements, the temperature was $294 \mathrm{~K}$, resulting in a vapor pressure of acetone of 192 Torr [23], and the atmospheric pressure was 761 Torr giving an acetone concentration of approximately 201,000 ppm. For ethanol the corresponding temperature and atmospheric pressure were $297 \mathrm{~K}$ and 747 Torr, resulting in a vapor pressure of ethanol of 55.3 Torr [24] and an ethanol concentration of $69,000 \mathrm{ppm}$. During the methanol measurements,

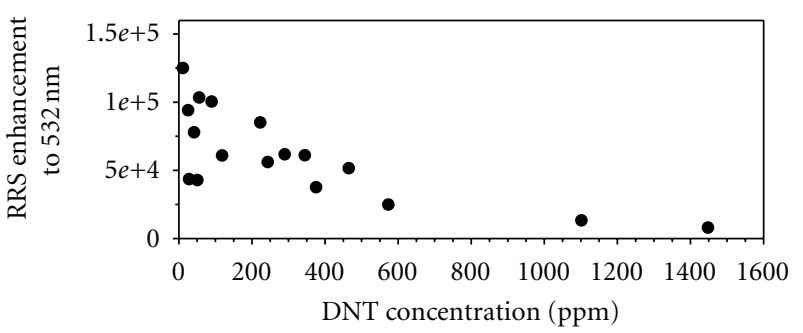

(a)

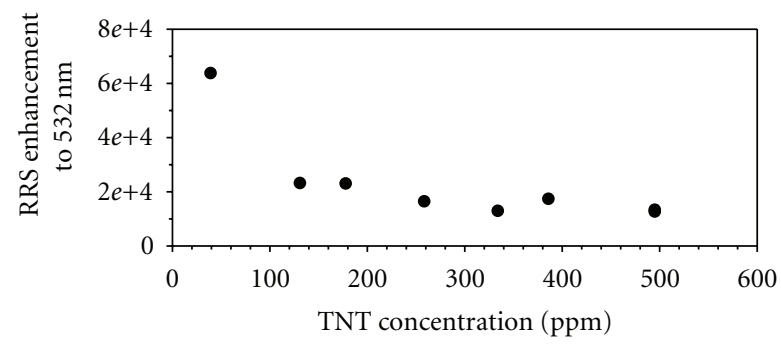

(b)

Figure 7: Apparent resonance enhancement of the Raman signal compared to the Raman cross section at $532 \mathrm{~nm}$ for (a) the $1347 \mathrm{~cm}^{-1}$ line in 2,4-DNT at different DNT concentrations and (b) the $1358 \mathrm{~cm}^{-1}$ line in 2,4,6-TNT at different TNT concentrations. The wavelength of the incident light was $248 \mathrm{~nm}$, and a $248 \mathrm{~nm}$ long-pass filter was used in both measurements. The enhancement varies with concentration due to the absorption effects. Note that the maximum enhancement for the higher concentrations of DNT is found at longer wavelengths than $248 \mathrm{~nm}$, whereas it is close to the wavelength giving maximum enhancement for TNT at $341 \mathrm{ppm}$.

the temperature was $297 \mathrm{~K}$, and the atmospheric pressure 747 Torr, resulting in a methanol vapor pressure of 120 Torr [25] and a methanol concentration of $138,000 \mathrm{ppm}$. For the measurements on a combination of solvent and NM, the concentrations were similarly determined: acetone and $\mathrm{NM}$ to $218,000 \mathrm{ppm}$ and $33,700 \mathrm{ppm}$, respectively, ethanol and NM to $66,200 \mathrm{ppm}$ and $40,400 \mathrm{ppm}$, respectively, and methanol and NM to $133,000 \mathrm{ppm}$ and $37,500 \mathrm{ppm}$, respectively.

Acetone, ethanol, and methanol all show a clear Raman spectrum at $232 \mathrm{~nm}$ at the given vapor concentrations. The lines are not overlapping the lines of NM, and there is no strong fluorescence from the solvents. 


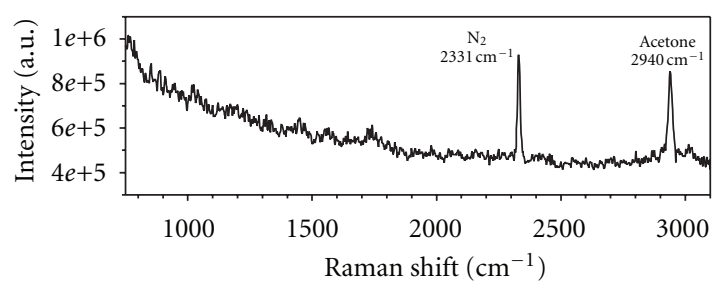

(a)

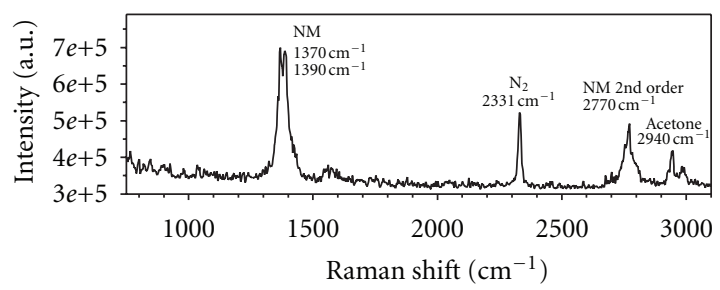

(b)

Figure 8: Raman signal from vapor phase measurements at a laser wavelength of $232 \mathrm{~nm}$ on (a) acetone at a concentration of 201,000 ppm and (b) acetone and NM at concentrations of $218,000 \mathrm{ppm}$ and $33,700 \mathrm{ppm}$, respectively.

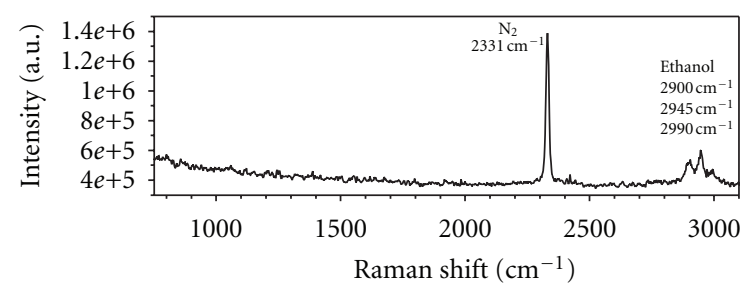

(a)

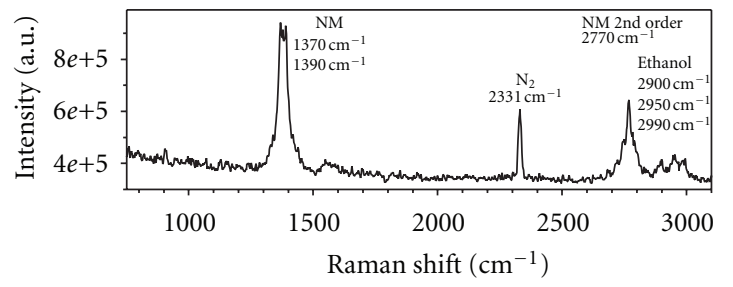

(b)

FIGURE 9: Raman signal from vapor phase measurements at a laser wavelength of $232 \mathrm{~nm}$ on (a) ethanol at a concentration of $69,000 \mathrm{ppm}$ and (b) ethanol and NM at concentrations of $66,200 \mathrm{ppm}$ and $40,400 \mathrm{ppm}$, respectively.

4.4. Outdoor Standoff Measurements on NM. Resonance Raman measurements were also made on NM in vapor phase outdoors, with the sample slightly heated and completely exposed to the wind. Figure 11 shows the NM spectrum measured outdoors at an irradiation wavelength of $220 \mathrm{~nm}$. The concentration of NM was not determined and therefore the cross section could not be determined. At $2331 \mathrm{~cm}^{-1}$, the $\mathrm{N}_{2}$ peak is seen, and the peak at about $1550 \mathrm{~cm}^{-1}$ originates from $\mathrm{O}_{2}$. The first order NM double peak is seen at approximately $1380 \mathrm{~cm}^{-1}$ and the second order Raman peak can be seen at about $2770 \mathrm{~cm}^{-1}$. In the outdoor measurements, it was possible to see the signal from NM vapor at shorter

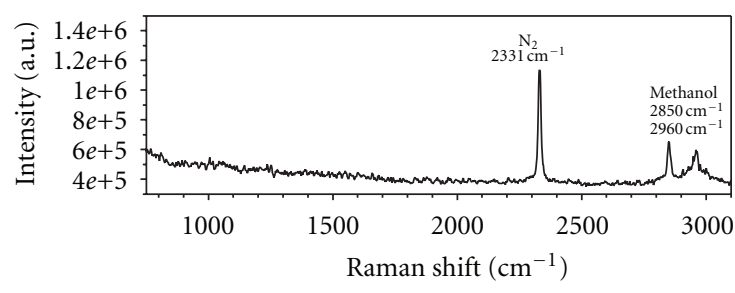

(a)

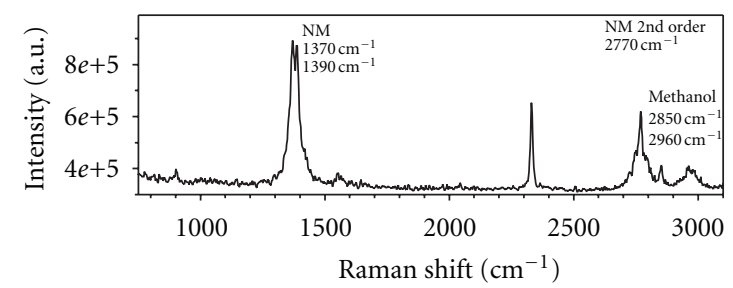

(b)

FIgURE 10: Raman signal from vapor phase measurements at a laser wavelength of $232 \mathrm{~nm}$ on (a) methanol at a concentration of $138,000 \mathrm{ppm}$ and (b) methanol and NM at concentrations of $133,000 \mathrm{ppm}$ and 37,500 ppm, respectively.

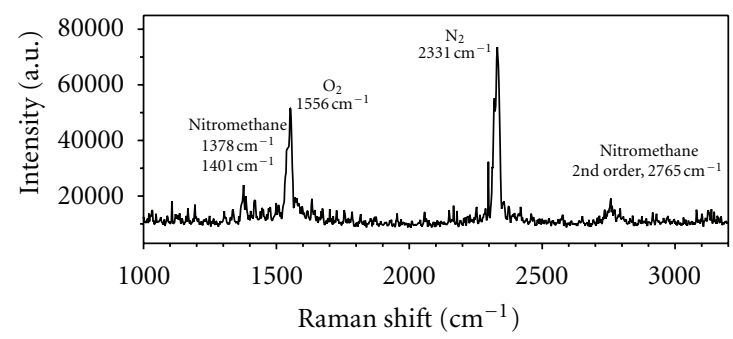

Figure 11: Resonance Raman spectrum from outdoor measurement on nitro methane in vapor phase at an irradiation wavelength of $220 \mathrm{~nm}$. The sample temperature was approximately $328 \mathrm{~K}$, the outdoor temperature was $274 \mathrm{~K}$, and the atmospheric pressure was about 755 Torr. The spectrum was accumulated during 1000 laser pulses.

wavelengths than was possible in the vapor chamber. The best signal was obtained at about $220 \mathrm{~nm}$ outdoors instead of at $232 \mathrm{~nm}$ as in the laboratory measurements. This is probably a result of a much lower NM concentration in the outdoor measurements, resulting in less absorption and selfabsorption and making it possible to measure the signal closer to the absorption peak.

\section{Conclusions}

Resonance Raman spectroscopy measurements have been performed on NM, DNT, and TNT in vapor phase at standoff distances. In addition, the Raman spectra of acetone, ethanol, and methanol in vapor phase were measured at $232 \mathrm{~nm}$. It was demonstrated that there is a clear enhancement of specific Raman lines in the explosive substances at UV wavelengths and that they can be used to identify vapor at a distance. The resonance enhancement for the explosive substances at different concentrations and wavelengths has 
been estimated to be between 10,000 and 250,000 compared to the Raman cross section at $532 \mathrm{~nm}$. The apparent cross sections and enhancements are reduced by absorption and self-absorption in the sample vapor, and the sensitivity is affected by the small size of the illuminated volume. It is believed that an increased volume and lower concentration in open air will have a positive effect on the sensitivity. Measurements performed on NM outdoors at $13 \mathrm{~m}$ distance under more realistic conditions showed good results, supporting this idea, and together with the laboratory results, they demonstrate the capability of resonance-enhanced Raman spectroscopy as a method to detect small amounts of explosives in vapor phase and its potential for detection of hidden explosives at stand-off distances.

\section{Acknowledgment}

The presented work has been funded by the Defense Advanced Research Projects Agency (DARPA).

\section{References}

[1] D. Menning and H. Ostmark, "Detection of liquid and homemade explosives: what do we need to know about their properties?" in Proceedings of the NATO Advanced Research Workshop on Detection of Liquid Explosives and Flammable Agents in Connection with Terrorism, pp. 55-70, St Petersburg, Russia, 2007.

[2] S. Wallin, A. Pettersson, H. Östmark, and A. Hobro, "Laserbased standoff detection of explosives: a critical review," Analytical and Bioanalytical Chemistry, vol. 395, no. 2, pp. 259-274, 2009.

[3] J. L. Gottfried, F. C. de Lucia, C. A. Munson, and A. W. Miziolek, "Laser-induced breakdown spectroscopy for detection of explosives residues: a review of recent advances, challenges, and future prospects," Analytical and Bioanalytical Chemistry, vol. 395, no. 2, pp. 283-300, 2009.

[4] N. R. Butt, M. Nilsson, A. Jakobsson et al., "Classification of raman spectra to detect hidden explosives," IEEE Geoscience and Remote Sensing Letters, vol. 8, pp. 517-521, 2010.

[5] S. K. Sharma, A. K. Misra, and B. Sharma, "Portable remote Raman system for monitoring hydrocarbon, gas hydrates and explosives in the environment," Spectrochimica Acta A, vol. 61, no. 10, pp. 2404-2412, 2005.

[6] J. C. Carter, S. M. Angel, M. Lawrence-Snyder, J. Scaffidi, R. E. Whipple, and J. G. Reynolds, "Standoff detection of high explosive materials at 50 meters in ambient light conditions using a small Raman instrument," Applied Spectroscopy, vol. 59, no. 6, pp. 769-775, 2005.

[7] A. Pettersson, I. Johansson, S. Wallin, M. Nordberg, and H. Östmark, "Near real-time standoff detection of explosives in a realistic outdoor environment at $55 \mathrm{~m}$ distance," Propellants, Explosives, Pyrotechnics, vol. 34, no. 4, pp. 297-306, 2009.

[8] A. Pettersson, S. Wallin, H. Östmark et al., "Explosives standoff detection using Raman spectroscopy: from bulk towards trace detection," in Proceedings of the Detection and Sensing of Mines, Explosive Objects, and Obscured Targets XV, R. S. Harmon, J. H. Holloway Jr., and J. T. Broach, Eds., vol. 7664, April 2010.

[9] M. Nordberg, F. Akke, and A. Pettersson, "Detection limits of stand-off spontaneous Raman scattering," Tech. Rep., Swedish Defence Research Agency, Stockholm, Sweden, 2009.
[10] H. S. Sands, I. P. Hayward, T. E. Kirkbride, R. Bennett, R. J. Lacey, and D. N. Batchelder, "UV-excited resonance Raman spectroscopy of narcotics and explosives," Journal of Forensic Sciences, vol. 43, no. 3, pp. 509-513, 1998.

[11] M. Gaft and L. Nagli, "UV gated Raman spectroscopy for standoff detection of explosives," Optical Materials, vol. 30, no. 11, pp. 1739-1746, 2008.

[12] N. A. Szymanski, Raman Spectroscopy: Theory and Practice, Plenum, New York, NY, USA, 1967.

[13] L. Nagli, M. Gaft, Y. Fleger, and M. Rosenbluh, "Absolute Raman cross-sections of some explosives: trend to UV," Optical Materials, vol. 30, no. 11, pp. 1747-1754, 2008.

[14] D. D. Tuschel, A. V. Mikhonin, B. E. Lemoff, and S. A. Asher, "Deep ultraviolet resonance raman excitation enables explosives detection," Applied Spectroscopy, vol. 64, no. 4, pp. 425-432, 2010.

[15] H. Östmark et al., "Vapor pressure of explosives: a critical review," Propellants, Explosives, Pyrotechnics. In press.

[16] R. B. Cundall, T. F. Palmer, and C. E. C. Wood, "Vapour pressure measurements on some organic high explosives," Journal of the Chemical Society, Faraday Transactions I, vol. 74, pp. 1339-1345, 1978.

[17] J. P. McCullough, D. W. Scott, R. E. Pennington, I. A. Hossenlopp, and G. Waddington, "Nitromethane: the vapor heat capacity, heat of vaporization, vapor pressure and gas imperfection; the chemical thermodynamic properties from 0 to $1500^{\circ} \mathrm{K}$," Journal of the American Chemical Society, vol. 76, no. 19, pp. 4791-4796, 1954.

[18] P. A. Pella, "Measurement of vapor pressures of TNT, 2,4-DNT 2,6-DNT, and EGDN," The Journal of Chemical Thermodynamics, vol. 9, no. 4, pp. 301-305, 1977.

[19] G. Edwards, "The vapor pressure of 2-4-6-trinitrotoluene," Transactions of the Faraday Society, vol. 46, pp. 423-427, 1950.

[20] H. Inaba and T. Kobayasi, "Laser-Raman radar-Laser-Raman scattering methods for remote detection and analysis of atmospheric pollution," Optical and Quantum Electronics, vol. 4, no. 2, pp. 101-123, 1972.

[21] M. J. Frisch, G. W. Trucks, and H. B. Schlegel, Gaussian 03, Revision C.02, Gaussian, Wallingford, Conn, USA, 2004.

[22] M. Gaft and L. Nagli, "Standoff laser based spectroscopy for explosives detection," in Electro-Optical Remote Sensing, Detection, and Photonic Technologies and Their Applications, vol. 6739 of Proceedings of SPIE, p. 673903, Florence, Italy, September 2007.

[23] D. Ambrose, C. H. S. Sprake, and R. Townsend, "Thermodynamic properties of organic oxygen compounds XXXIII. The vapour pressure of acetone," The Journal of Chemical Thermodynamics, vol. 6, no. 7, pp. 693-700, 1974.

[24] C. B. Kretschmer and R. Wiebe, "Liquid-vapor equilibrium of ethanol-toluene solutions," Journal of the American Chemical Society, vol. 71, no. 5, pp. 1793-1797, 1949.

[25] D. Ambrose and C. H. S. Sprake, "Thermodynamic properties of organic oxygen compounds XXV. Vapour pressures and normal boiling temperatures of aliphatic alcohols," The Journal of Chemical Thermodynamics, vol. 2, no. 5, pp. 631-645, 1970. 


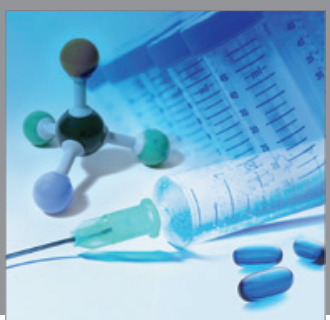

International Journal of

Medicinal Chemistry

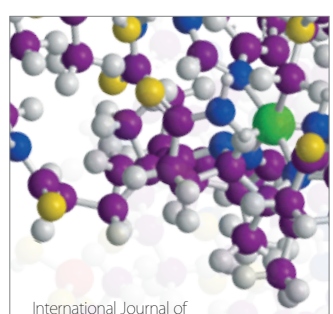

Carbohydrate Chemistry

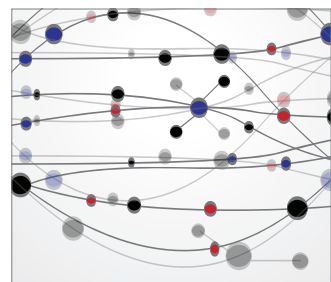

The Scientific World Journal
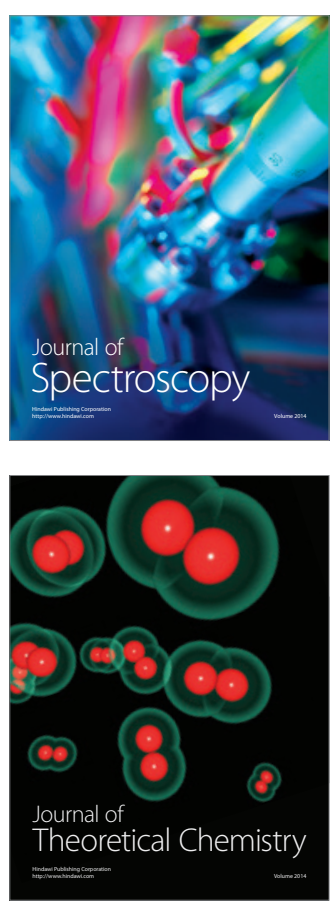
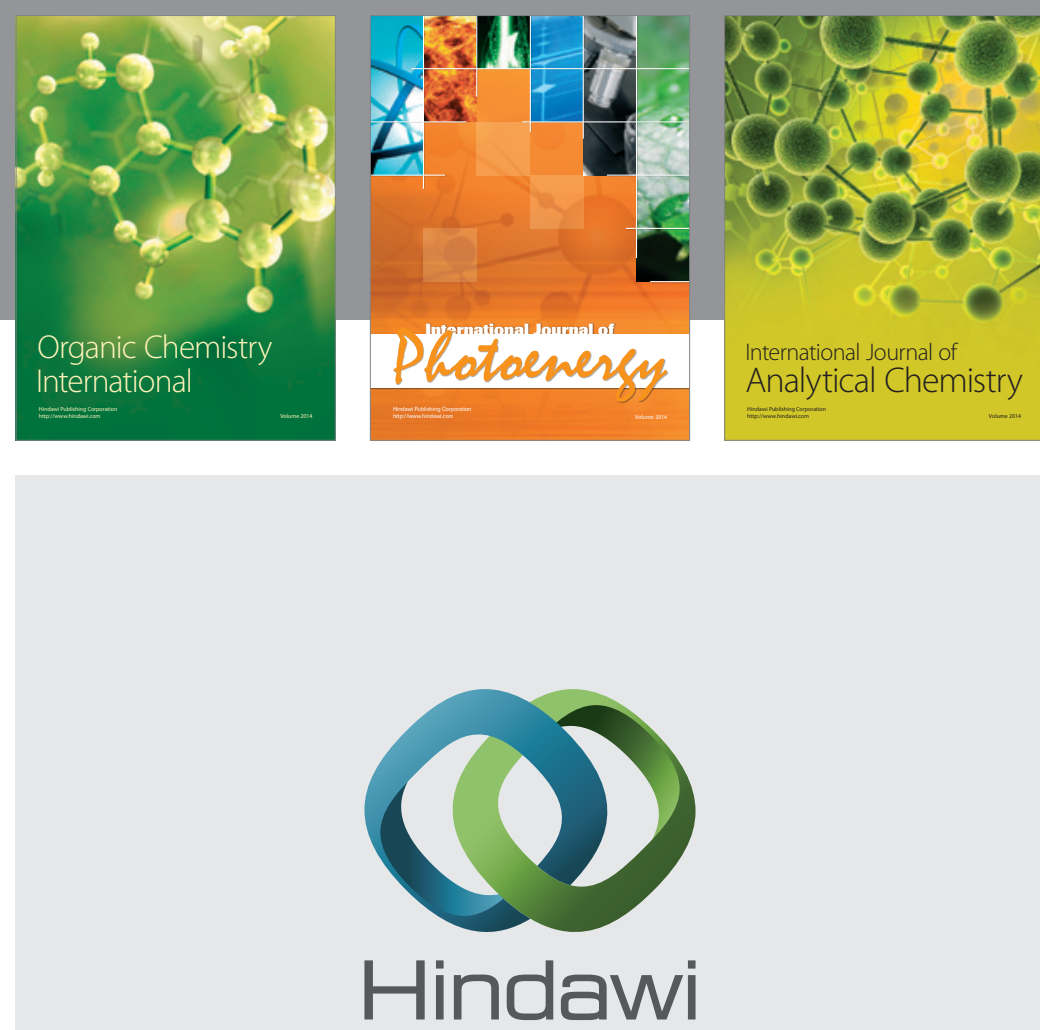

Submit your manuscripts at

http://www.hindawi.com
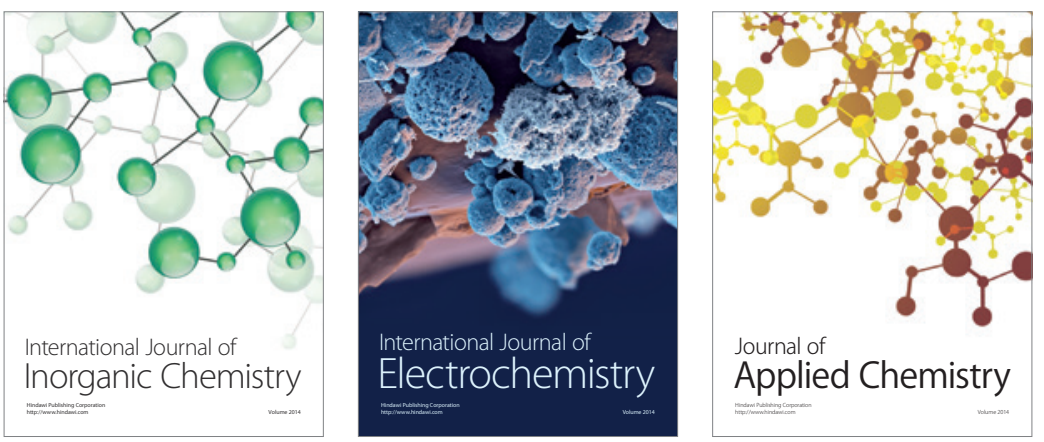

Journal of

Applied Chemistry
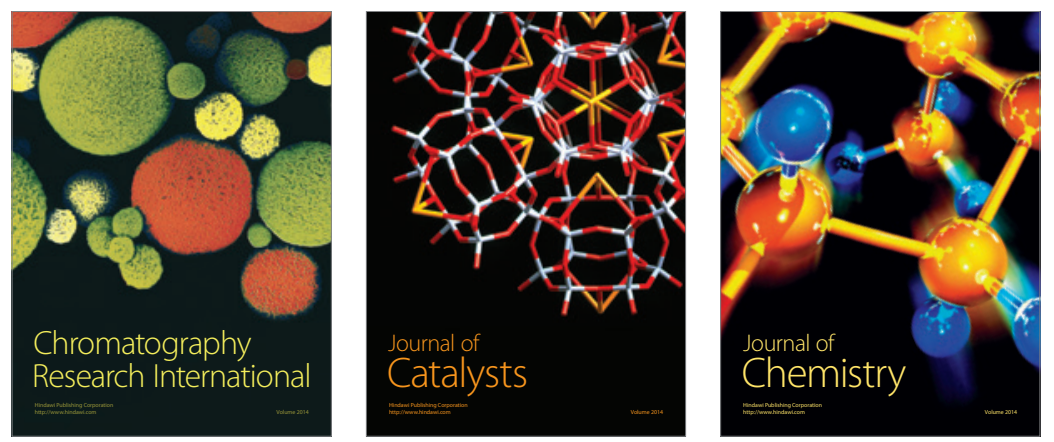
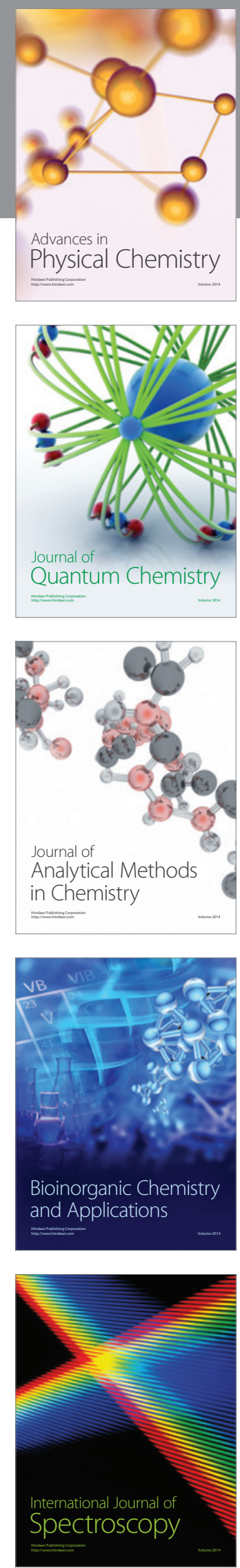